\title{
Recombinant BCG exporting ESAT-6 confers enhanced protection against tuberculosis
}

\author{
Alexander S. Pym ${ }^{1,2}$, Priscille Brodin ${ }^{1}$, Laleh Majlessi ${ }^{3}$, Roland Brosch ${ }^{1}$, \\ Caroline Demangel ${ }^{1}$, Ann Williams ${ }^{4}$, Karen E. Griffiths ${ }^{4}$, Gilles Marchal ${ }^{5}$, \\ Claude Leclerc $^{3} \&$ Stewart T. Cole $^{1}$ \\ ${ }^{1}$ Unité de Génétique Moléculaire Bactérienne, Institut Pasteur, Paris, France \\ ${ }^{2}$ Liverpool School of Tropical Medicine, Liverpool, UK \\ ${ }^{3}$ Unité de Biologie des Régulations Immunitaires, INSERM E0352, Institut Pasteur, Paris, France \\ ${ }^{4}$ Centre for Applied Microbiology and Research, Salisbury, UK \\ ${ }^{5}$ Laboratoire de Référence des Mycobactéries, Institut Pasteur, Paris, France \\ A.S.P., P.B. and L.M. contributed equally to this work. \\ Correspondence should be addressed to S.T.C.; e-mail: stcole@pasteur.fr
}

Published online 14 April 2003; doi:10.1038/nm859

\begin{abstract}
The live tuberculosis vaccines Mycobacterium bovis BCG (bacille Calmette-Guérin) and Mycobacterium microti both lack the potent, secreted T-cell antigens ESAT-6 (6-kDa early secretory antigenic target) and CFP-10 (10-kDa culture filtrate protein). This is a result of independent deletions in the region of deletion-1 (RD1) locus, which is intact in virulent members of the Mycobacterium tuberculosis complex. To increase their immunogenicity and protective capacity, we complemented both vaccines with different constructs containing the es $X A$ and es $X B$ genes, which encode ESAT- 6 and CFP-10 respectively, as well as a variable number of flanking genes. Only reintroduction of the complete locus, comprising at least 11 genes, led to full secretion of the antigens and resulted in specific ESAT-6-dependent immune responses; this suggests that the flanking genes encode a secretory apparatus. Mice and guinea pigs vaccinated with the recombinant strain BCG::RD1-2F9 were better protected against challenge with $M$. tuberculosis, showing less severe pathology and reduced dissemination of the pathogen, as compared with control animals immunized with BCG alone.
\end{abstract}

The live, attenuated BCG vaccine, originally derived by serial passage of a virulent strain of $M$. bovis, has been used to prevent human tuberculosis since 1921. BCG is effective against severe forms of childhood tuberculosis but is of limited efficacy against adult pulmonary disease in endemic areas ${ }^{1}$. Several reasons for its variable efficacy have been proposed, ranging from the influence of prior infection with environmental mycobacteria ${ }^{2}$ to the absence of antigens that are protective against $M$. tuberculosis ${ }^{1,3}$. Comparative genomics identified $>100$ coding sequences absent from BCG but present in M. tuberculosis, the principal agent of human tuberculosis ${ }^{4-7}$, that were lost either during the evolutionary divergence of $M$. bovis from $M$. tuberculosis or during the in vitro passaging of BCG and its progenitor. Some of these coding sequences encode potential antigens that could improve immunogenicity if reintroduced into BCG.

Among the missing genes are the adjacent coding sequences es $x B$ and es $x A$, which respectively encode CFP-10 and ESAT-6, low-molecular-weight proteins that induce potent Th1 responses ${ }^{3,8-13}$. They elicit protection against tuberculosis in animal models when administered as subunit or DNA vaccines but are not superior to $\mathrm{BCG}^{14}$. Independent deletion events in the RD1 locus (Fig. 1) removed es $x B$, es $x A^{15}$ and flanking genes from BCG and the related live vaccine $M$. microti ${ }^{16,17}$. ESAT- 6 and CFP-10 are abundant in short-term culture filtrate but lack obvious secretion signals, implying Sec-independent transport from the cy- tosol. The es $x B$ and es $x A$ genes are part of the es $x A-V$ gene family, with 23 members in $M$. tuberculosis. These are usually arranged as couplets in an operon. In five instances, including the RD1 locus, the es $x$ genes are part of a conserved segment that arose by duplication, encoding members of five additional protein families ${ }^{18-20}$. The precise function of most of the coding sequences in these regions is unknown, but their linkage with es $x$ genes suggests they could encode a protein complex required for the export of ESAT- 6 proteins ${ }^{18-20}$.

Knowledge of antigen secretion is crucial for the development of recombinant BCG vaccines. The observation that only live BCG elicits substantial protection indicates that transport of antigenic proteins to the cell wall and beyond is essential for maximizing protective immune responses against tuberculosis. Many of the most promising candidates for vaccine development are secreted proteins ${ }^{21-23}$. As a first step to constructing a recombinant BCG vaccine expressing ESAT-6 and CFP-10, we adopted a combinatorial complementation strategy to identify which genes were essential for antigen export and showed that the entire gene cluster is required for maximal ESAT-6 delivery from the cytosol.

\section{General organization of esx loci and secretion}

Advanced bioinformatic analysis of the esx loci has been described previously ${ }^{18,19}$ and subsequently expanded ${ }^{20,24}$. Briefly, 11 


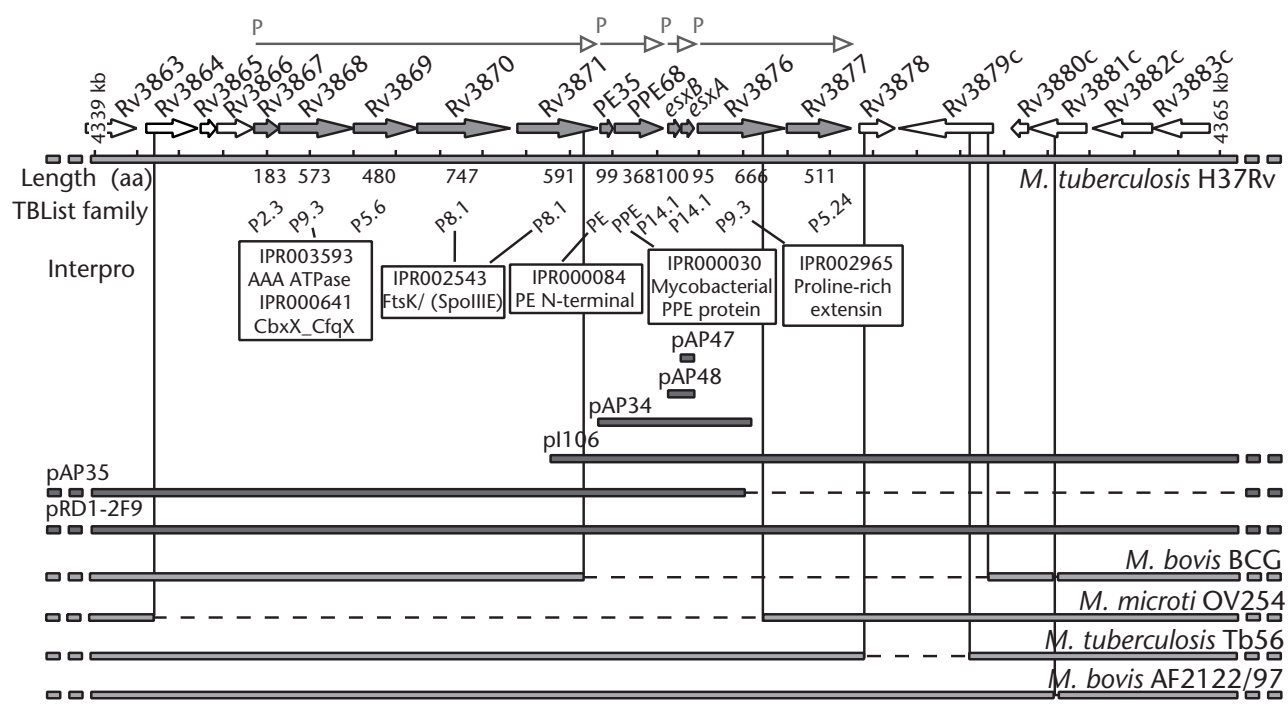

Fig. 1 Diagram of the M. tuberculosis H37Rv genomic region showing the positions of various genes, probable transcriptional units and constructs used. Also shown is a map of fragments used to complement BCG and M. microti and genomic regions deleted from different mycobacterial strains. The middle part shows key genes, putative promoters $(P)$ and possible transcripts, various proteins from the RD1 region and their sizes (number of amino acid residues), InterPro domains (http://www.ebi.ac.uk/interpro/) and membership of $M$. tuberculosis protein families according to TubercuList (TBList; http://genolist.pasteur.fr/TubercuList/). The dashed lines mark the extent of the RD1 deletion in BCG, M. microti and M. tuberculosis clinical isolate MT56 (ref. 28). M. bovis AF2122/97 is shown because like BCG, it contains a frameshift mutation in Rv3881, a gene flanking the RD1 region of BCG. The fragments are drawn to show their ends in relation to the genetic map unless they extend beyond the genomic region indicated. The pRD1-2F9, pRD1-1106 and pAP35 constructs are based on pYUB412, pAP34 on pKINT, and pAP47 and pAP48 on pSM81.

loci were identified that contained es $x$ couplets; 5 of these show an extended configuration. Four transcriptional units encompassing the esxBA cluster (Rv3867-Rv3877) were defined in silico (Fig. 1); some experimental support for these is available ${ }^{8}$. Predicted functions for the first unit (Rv3867-Rv3871) include a membrane-associated protein (Rv3869), an ATP-dependent chaperone of the AAA-family (Rv3868 (ref. 25)) and a dimeric membrane-bound ATPase of the FtsK (SpoIIIE) family (Rv3870 and Rv3871 (ref. 24)). The second and third units encode the PE and PPE proteins (with their characteristic N-terminal motifs pro-glu and pro-pro-glu and the two ESAT-6 proteins, respectively, while the fourth unit codes for a proline-rich polypeptide (Rv3876) related to the putative chaperone and an integral membrane protein with 11 membrane-spanning helices (Rv3877). ESAT-6 and CFP-10 form a heterodimer ${ }^{26}$ that may require chaperone activity, possibly effected by the ATP-dependent protein clamp Rv3868 (ref. 25). Alternatively, chaperones may prevent premature protein-protein interactions as in type III secretion system ${ }^{27}$. This functional information and the tight linkage of cognate genes with es $x$ members suggested that some or all of these proteins are required for ESAT-6 secretion. Experimental support for much of this working model is provided below

\section{Complementation of the RD1 locus of BCG and M. microti}

The RD1 deletion in BCG disrupts nine coding sequences and affects all four transcriptional units. Three are removed entirely, while the fourth (Rv3867-Rv3871) is intact apart from the loss of 112 codons from the $3^{\prime}$ end of Rv3871 (Fig. 1). Transcriptome analysis of BCG using DNA microarrays indicated that the Rv3867-Rv3871 transcriptional unit was still active. The RD1 ${ }^{\text {mic }}$ deletion removes three transcriptional units completely, with only Rv3877 remaining from the fourth. M. tuberculosis clinical isolate MT56 does not have Rv3878 or Rv3879 (ref. 28) but still secretes ESAT- 6 and CFP-10, whereas BCG and M. bovis have frameshifts in Rv3881 (Fig. 1).

To determine whether a dedicated export machinery exists and to establish which genes are essential for a vaccine strain to secrete ESAT-6 and CFP-10, we assembled a series of clones that stably insert into the M. tuberculosis attB site. The pAP34 clone carries only the antigenic core region encoding ESAT-6, CFP-10 and the upstream $P E$ and $P P E$ genes, whereas the RD1-I106 and RD1-pAP35 clones include the core region and either the downstream or upstream portion of the gene cluster (Fig. 1). The RD1-2F9 construct contains a 32-kb segment (Rv3861-Rv3885) covering the entire RD1 gene cluster. We chose large genomic fragments to avoid polar effects that might be expected if a putative protein complex is only partially complemented in trans. We also established expression constructs (pAP47 and pAP48) in which genes are transcribed from a heat-shock promoter.

\section{Genes required for export of ESAT-6 and CFP-10}

Four BCG-RD1 recombinants (BCG::RD1-pAP34, BCG::RD1pAP35, BCG::RD1-2F9 and BCG::RD1-I106; Fig. 2) were initially tested for correct expression of ESAT-6 and CFP-10.

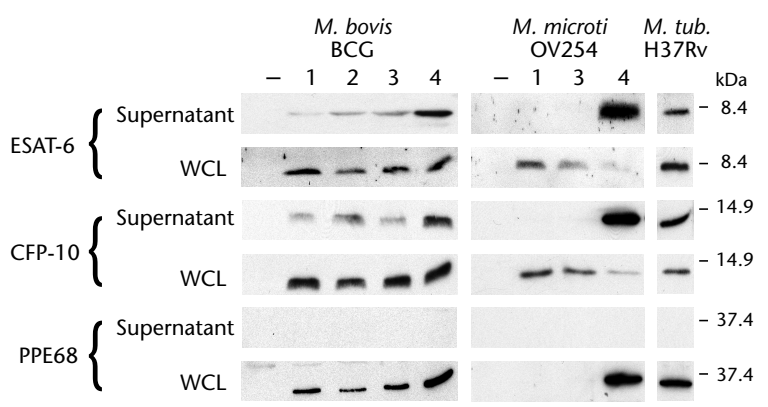

Fig. 2 Western blot analysis of various RD1 knock-ins of $M$. bovis BCG and M. microti. Left, results of immunodetection of ESAT-6, CFP-10 and PPE68 (Rv3873) in whole-cell lysates (WCL) and culture supernatants of BCG recombinants; center, equivalent findings from M. microti; right, M. tuberculosis (M. tub. H37Rv) control samples. Samples were obtained from mycobacteria, transformed with various plasmids, grown in parallel. Equal quantities of protein were present in each of the lanes. Lane numbers correspond to the following: -, pYUB412 vector control; 1, pAP34; 2, pAP35; 3, RD1-I106; 4, RD1-2F9. The positions of the nearest molecular weight markers are indicated. 
Immunoblotting of whole-cell protein extracts from mid-log phase cultures of the various recombinants using a monoclonal antibody against ESAT-6 (ref. 29) or polyclonal sera against CFP-10 and the PPE protein Rv3873 (ref. 30) showed that all three proteins were expressed at levels comparable to those in M. tuberculosis (Fig. 2). Although low levels of ESAT-6 and CFP-10 were detected in the concentrated supernatant protein fractions of these recombinants, it was only with the construct encompassing the entire es $x$ gene cluster (BCG:: RD1-2F9) that the two antigens accumulated in substantial amounts. The high concentrations seen in the supernatant of the recombinant BCG::RD1-2F9 were not due to a non-specific increase in permeability or loss of cell wall material, because when the same protein fractions were immunoblotted for Rv3873, this protein was only detected in the cell wall. Furthermore, with pAP47 and pAP48, which contained es $x A$ or es $x B A$ alone, ESAT- 6 did not accumulate in the culture supernatant (data not shown).

To assess the effect of the RD1 ${ }^{\text {mic }}$ deletion on antigen handling, the experiments were replicated in M. microti. As with BCG, ESAT-6 and CFP-10 were only exported in substantial amounts when expressed in conjunction with the entire es $x$ cluster (Fig. 2). Exclusively cellular expression was also seen with the M. microti RD1-pAP35 recombinant strain (data not shown). Although it is possible that the increase in supernatant antigens seen with BCG::RD1-2F9 could be caused by changes in expression, the combined findings suggest that complementation with esxA or esx $B$ alone is insufficient to produce a recombinant vaccine that secretes these antigens. Rather, secretion requires expression of genes located both upstream and downstream of the antigenic core region, confirming our hypothesis ${ }^{19}$ that the conserved es $x$ gene cluster does indeed encode functions essential for antigen export.

\section{Specific T-cell responses require ESAT- 6 secretion}

Since the classical observation that inoculation with live, but not killed, BCG confers protection against tuberculosis, the secretion of antigens has been considered crucial for maximizing protective T-cell immunity. We therefore tested whether antigen secretion is essential for eliciting ESAT6-specific T-cell responses. Groups of C57BL/6 mice were subcutaneously inoculated with one of six recombinant vaccines (BCG- pAP47, BCG-pAP48, BCG::RD1-pAP34, BCG::RD1-pAP35, BCG:: RD1-I106 or BCG::RD1-2F9) or with BCG transformed with the empty vector pYUB412. Three weeks after vaccination, we assessed T-cell immunity by comparing antigen-specific splenocyte proliferation and interferon (IFN)- $\gamma$ production (Fig. 3). As anticipated, all seven vaccines induced splenocyte proliferation and IFN- $\gamma$ production in response to partially purified protein derivative (PPD) but not a control peptide (Mal-E), indicating successful vaccination. However, only splenocytes from the mice inoculated with BCG::D1-2F9 markedly proliferated and produced IFN- $\gamma$ in response to the immunodominant ESAT-6 peptide (Fig. 3) or recombinant CFP-10 protein (data not shown). These data show that export of antigens is essential for stimulating specific Th1-oriented T cells.

To further characterize the immune response, we immunized mice with BCG::RD1-2F9 or control BCG. Splenocytes in both
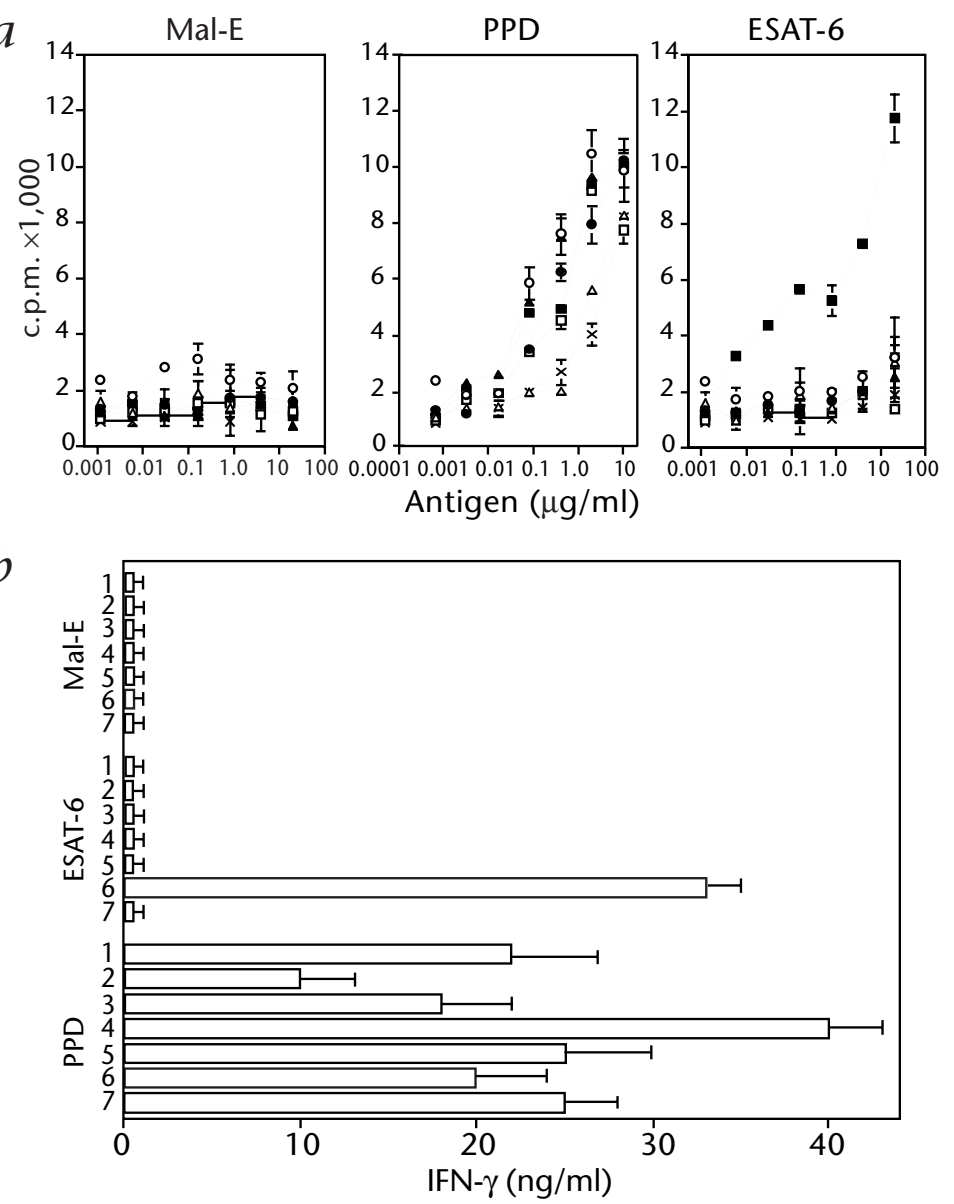

Fig. 3 Analysis of immune responses induced by the BCG recombinants. $\boldsymbol{a}$, Results of splenocyte proliferation assays in response to in vitro stimulation with a peptide from Mal-E (negative control), PPD or a peptide containing an immunodominant epitope from ESAT-6 after vaccination with each of the recombinants. $\bigcirc$, BCG 06; $\mathbf{\square}, \mathrm{BCG}: \mathrm{RD} 1-2 \mathrm{~F} 9 ; \times, \mathrm{BCG}: \mathrm{:pYUB} 412 . \boldsymbol{b}, \mathrm{IFN}-\gamma$ production by splenocytes in to the same antigens as in (a) for each of the various BCG transformants. Ba BCG-pAP47; bar 2, BCG-pAP48; bar 3, BCG::RD1-pAP34; bar 4, BCG::RD1-pAP35 ar 5, BCG::RD1-1106; bar 6, BCG::RD1-2F9; bar 7, BCG-pYUB412.

mice proliferated in response to the immunodominant antigen 85A peptide (Fig. 4a). The strong splenocyte proliferation in the presence of ESAT- 6 was abolished by a CD4-specific, but not a CD8-specific, monoclonal antibody, indicating that the $\mathrm{CD} 4^{+} \mathrm{T}$ cell subset was involved (Fig. $4 \mathrm{~b}$ ). In vitro IFN- $\gamma$ response to PPD and the ESAT peptide suggested that subcutaneous immunization generated stronger T-cell responses (Fig. 4c) compared with intravenous injection. These results show that the ESAT-6 T-cell immune response to vaccination with BCG::RD1-2F9 is potent, reproducible and robust, making this recombinant an excellent candidate for protection studies.

Protective efficacy of BCG::RD1-2F9 in immunocompetent mice When used alone as a subunit or DNA vaccine, ESAT-6 induces weaker protection against tuberculosis than does $\mathrm{BCG}^{14}$. Thus, it was of interest to determine whether the presentation of ESAT-6 or CFP-10 to the immune system by BCG, mimicking natural infection, could increase protection. Groups of C57BL/6 mice were inoculated intravenously with BCG::RD1-2F9 or BCG::pYUB412 and challenged intravenously eight weeks later with $M$. tubercu- 
$a$

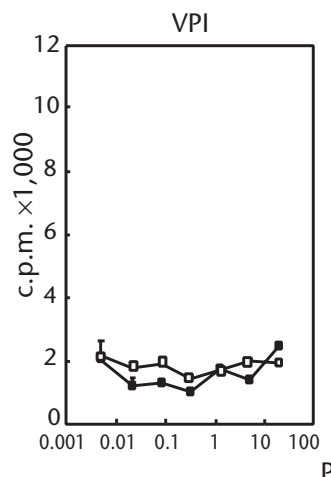

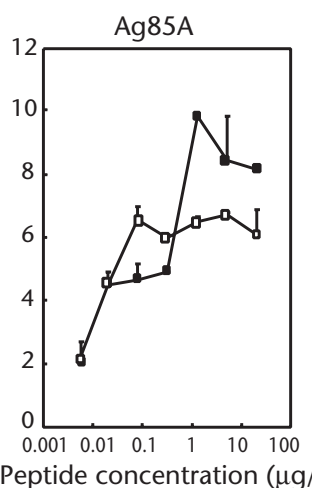

Peptide concentration $(\mu \mathrm{g} / \mathrm{ml})$

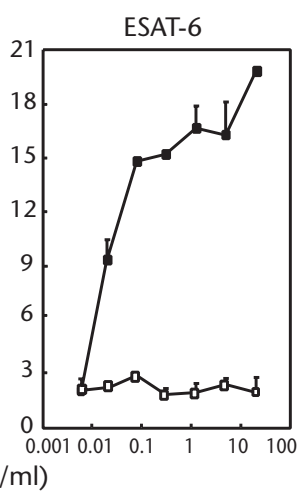

$b$

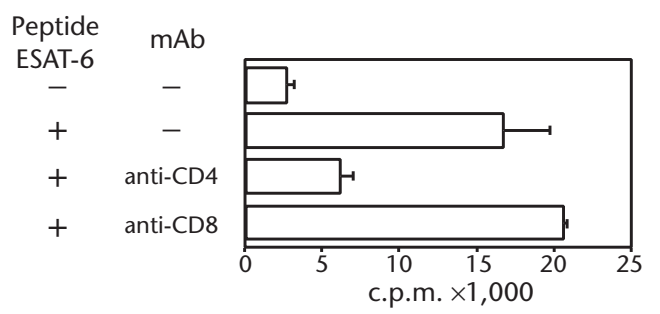

Fig. 4 Further immunological characterization of responses to BCG::RD1-2F9. $\boldsymbol{a}$, Proliferative response of splenocytes of $\mathrm{C} 57 \mathrm{BL} / 6$ mice immunized subcutaneously with $10^{6}$ CFU of BCG::pYUB412 ( $\square$ ) or BCG::RD1-2F9 (回) to in vitro stimulation with various concentrations of synthetic peptides from poliovirus type-1 capsid protein VP1 (negative control), antigen 85A or ESAT-6 (see Methods for details). b, Proliferation of splenocytes from BCG::RD12F9-immunized mice in the absence or presence of ESAT-6 peptide, with or without monoclonal antibodies (mAb) against CD4 or CD8. Results are expressed as mean \pm s.d. of $\left[{ }^{3} \mathrm{H}\right]$ thymidine incorporation from duplicate wells. c, Concentration of IFN- $\gamma$ in culture supernatants of splenocytes of $\mathrm{C} 57 \mathrm{BL} / 6$ mice stimulated for $72 \mathrm{~h}$ with peptides or PPD after subcutaneous or intravenous immunization with either BCG::pYUB412 (106 CFU, $\square)$, BCG::pYUB412 $\left(10^{7} \mathrm{CFU}, \square\right), \mathrm{BCG}: \mathrm{RD} 1-2 \mathrm{F9}$ (10 $\left.\mathrm{CFU}, \mathbf{\square}\right)$ or BCG::RD1-2F9 (10 $\left.7 \mathrm{CFU}^{-} \square\right)$.

\section{C}

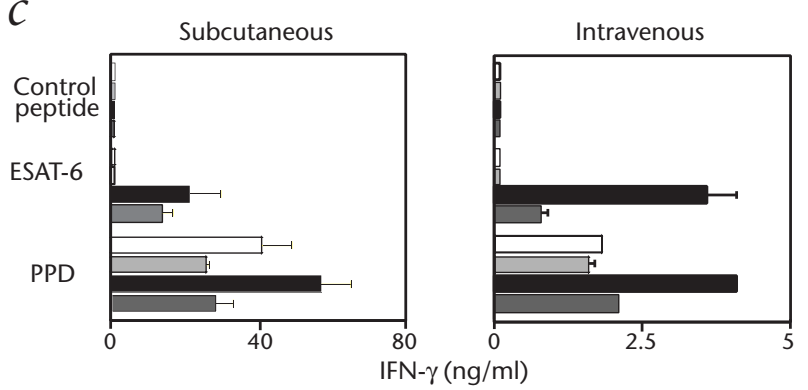
Results are expressed as the mean \pm s.d. of duplicate culture wells.

losis H37Rv. Growth of M. tuberculosis in spleen and lungs of each vaccinated cohort was compared with that of unvaccinated controls two months after infection. Organ homogenates for bacterial enumeration were plated on 7H11 medium, with or without hygromycin, to differentiate $M$. tuberculosis from residual BCG colonies (Fig. 5a). The results show that compared with BCG vaccination, the BCG::RD1-2F9 vaccine inhibited growth of $M$. tuberculosis H37Rv in the spleens by $0.4 \log _{10}$ colony-forming units (CFU) but was of comparable efficacy in the lungs.

We repeated the challenge experiment using the aerosol route. Antibiotic treatment was used to clear persisting BCG from mouse organs before infection with $M$. tuberculosis to ensure that any differences between BCG::RD1-2F9 and BCG were the result of the memory of the immune system and not of the increased persistence of this recombinant ${ }^{30}$. Two months after vaccination, C57BL/6 mice were treated with antibiotics for three weeks and then aerosol-infected with $100 \mathrm{CFU}$. Bacterial enumeration in the spleens and lungs showed that after respiratory infection, vaccination with BCG::RD1-2F9 was superior to vaccination with the control strain of BCG (Fig. $5 b$ ). Growth of M. tuberculosis, however, was again only inhibited strongly in the mouse spleens. In both of these mouse experiments, the levels of protection achieved by the control BCG strain were less than in some previously published studies, possibly reflecting variation attributable to differences between BCG daughter strains ${ }^{31}$.

Fig. 5 Mouse protection studies. $\boldsymbol{a}$, Bacterial counts in the spleen and lungs of vaccinated and unvaccinated C57BL/ 6 mice 2 months after intravenous challenge with $M$. tuberculosis H37Rv. Results are expressed as the mean \pm s.d. of 4 mice. $\boldsymbol{b}$, Bacterial counts in the spleen and lungs of vaccinated and unvaccinated $\mathrm{C} 57 \mathrm{BL} / 6$ mice after aerosol challenge with 100 CFU of $M$. tuberculosis. Data are the mean \pm s.d. of groups of 3 mice. $\square$, cohort of unvaccinated mice; $\square$, mice vaccinated with BCG::pYUB412; $\mathbf{\square}$, mice vaccinated with $B C G:: R D 1-2 F 9 .{ }^{*}, P<0.05 ;{ }^{* *}, P<0.01$.

\section{Protective efficacy of BCG::RD1-2F9 in guinea pigs}

Although experiments in mice showed the superior protective efficacy of BCG::RD1 over BCG, it was important to establish a similar effect in the guinea pig, a more appropriate model of human tuberculosis. Groups of six guinea pigs were inoculated subcutaneously with saline, BCG::RD1-2F9 or BCG and challenged eight weeks later with $M$. tuberculosis through the aerosol route. Individual guinea pigs were weighed weekly and

$$
a
$$

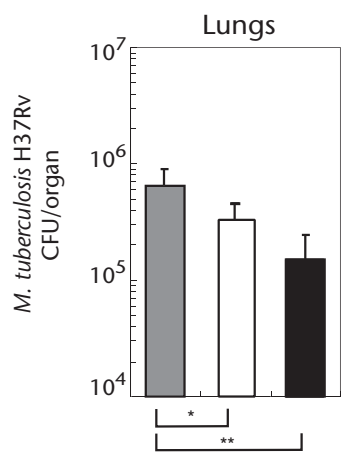

$b$

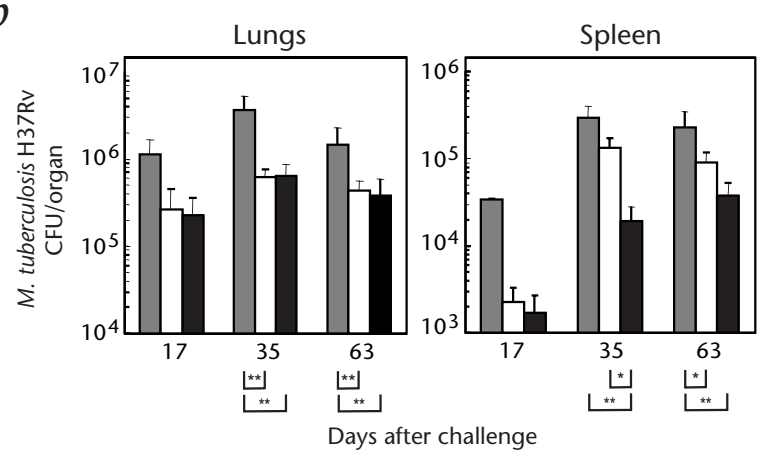


$a$

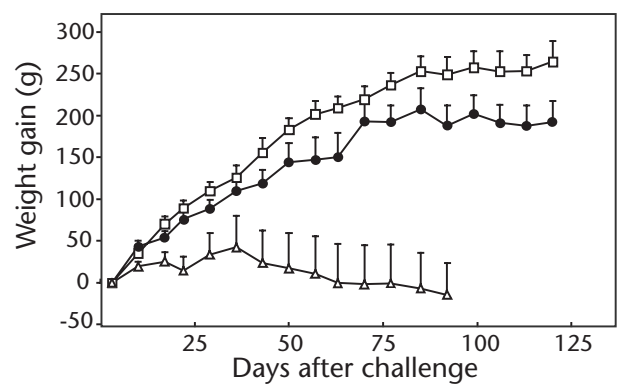

$b$

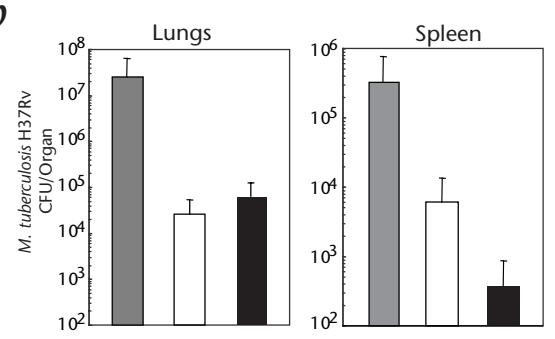

C

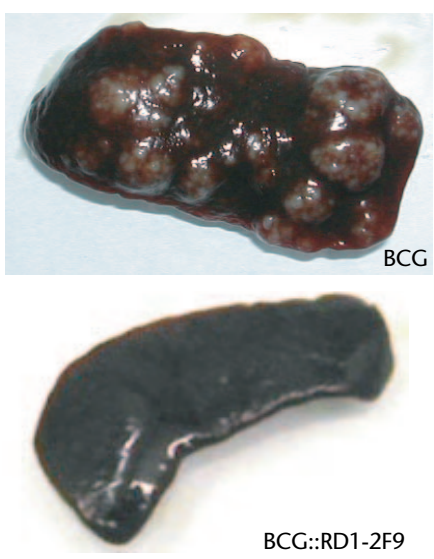

Fig. 6 Guinea pig protection studies. a, Mean weight gain of vaccinated and unvaccinated guinea pigs after aerosol infection with $M$. tuberculosis H37Rv. Guinea pigs were injected with saline $(\triangle)$, BCG $(\square)$ or BCG::RD1-2F9 (O). Error bars represent s.e.m. Each time point represents the mean weight of 6 guinea pigs. For the saline-injected group, the last live weight was used for calculating the mean, as the guinea pigs were killed on signs of severe tuberculosis (after 50, 59, 71, 72, 93 and 93 d). $\boldsymbol{b}$, Mean bacterial counts in the spleen and lungs of vaccinated and unvaccinated guinea pigs after aerosol challenge with $M$. tuberculosis H37Rv. Groups of 6 guinea pigs were injected subcutaneously with saline

( $\square)$, BCG ( $\square)$ or BCG::RD1-2F9 (ם) and infected $56 \mathrm{~d}$ later. Vaccinated guinea pigs were killed $120 \mathrm{~d}$ after infection, and unvaccinated ones on signs of suffering or significant weight loss. Error bars represent the s.e.m. of 6 guinea pigs. c, Spleens of vaccinated guinea pigs $120 \mathrm{~d}$ after infection with $M$. tuberculosis H37Rv. Guinea pigs were vaccinated with BCG (top) or BCG::RD1-2F9 (bottom).

killed 17 weeks after challenge, or earlier if signs of severe tuberculosis developed. Whereas all unvaccinated guinea pigs were euthanized before the last time-point because of overwhelming disease, both of the vaccinated cohorts progressively gained weight and remained clinically well (Fig. 6a). This indicated that although the BCG::RD1-2F9 recombinant is more virulent in severely immunodeficient mice $^{30}$, there is no increased pathogenesis in the highly susceptible guinea pig model of tuberculosis. When the bacterial loads in the spleens were compared, the number of CFU recovered from the guinea pigs immunized with BCG::RD1-2F9 was less than 10\% of that from guinea pigs immunized with BCG (Fig. $6 b$ ). This was significant using a one-sided Student's $t$-test $(P=0.05)$. There was no significant difference between the number of CFU in the lungs of the two vaccinated groups, indicating that the organspecific enhanced protection observed in mice vaccinated with BCG::RD1-2F9 also occurred in guinea pigs. The reduction of splenic bacterial loads was also reflected in the gross pathology. Visual examination showed that tubercles on the surface of spleens from BCG-vaccinated mice were much larger and more numerous than those from BCG::RD1-2F9-vaccinated guinea pigs (Fig. 6c).

\section{Discussion}

Reasons for the recalcitrance of the tuberculosis pandemic are multifactorial but include the modest efficacy of BCG. Two broad approaches can be used for the development of improved tuberculosis vaccines ${ }^{21,32,33}$ : the creation of subunit vaccines based on purified protein antigens or of new live vaccines that stimulate a wider range of immune responses. Although a growing list of individual or combination subunit vaccines and hybrid proteins has been tested, none has proved superior to BCG in animal models ${ }^{21}$. Similarly, new attenuated vaccines derived from virulent $M$. tuberculosis have yet to outperform $\mathrm{BCG}^{34,35}$. The only vaccine that appears to surpass BCG is a BCG recombinant overexpressing antigen $85 \mathrm{~A}^{23}$. The basis for developing this vaccine was the notion that overexpression of an immunodominant T-cell antigen could quantitatively enhance the BCGelicited immune response.
In this study, we adopted a different approach, exploiting previous comparative genomic studies ${ }^{4,5,15,36}$. We reasoned that BCG could be improved qualitatively by reintroducing lost $M$. tuberculosis antigens. Using complementation, we showed that restoration of the RD1 locus does improve the protective efficacy of BCG and defines a genetic modification that should be included in new recombinant BCG vaccines. In addition, two of our findings will be crucial for the development of live tuberculosis vaccines. First, we identified the genetic basis of secretion of the ESAT- 6 family of immunodominant T-cell antigens. Second, we showed that export of ESAT-6 proteins from the cytosol is essential for maximizing their antigenicity.

The extracellular proteins of M. tuberculosis are a rich source of protective antigens $s^{11,12,22,37}$. It is not known how some of these proteins, which lack conventional secretion signals, are exported from the cytosol. Although two secA orthologs were identified in the genome sequence of $M$. tuberculosis ${ }^{18}$, we did not detect any genes for obvious type I, II or III protein secretion systems, like those of Gram-negative bacterial pathogens ${ }^{38}$. This suggests that other secretion systems exist. An in silico analysis of the $M$. tuberculosis proteome identified a set of proteins and genes that could constitute such a system, based on their inferred functions, genomic organization and strict association with the es $x$ gene family ${ }^{19}$. Our results provide the first empirical evidence that this gene cluster is essential for the normal export of ESAT- 6 and CFP-10 and indicate that at least Rv3871 and either Rv3876 or Rv3877 are essential for the normal secretion of ESAT-6, as these are the only conserved genes disrupted in BCG that are not complemented by RD1-I106 or RD1-pAP35. The precise roles of the individual components of the ESAT- 6 secretory apparatus can now be addressed experimentally using the tools developed here.

The second major finding of this study is that the secretion of ESAT-6 (and probably CFP-10) is crucial for inducing maximal Tcell responses, although other RD1-encoded proteins (such as the PPE68 protein (Rv3873) located in the cell envelope ${ }^{30}$ ) may also contribute. We showed that only the vaccine strain exporting ESAT-6 through an intact secretory apparatus elicits powerful antigen-specific T-cell responses. Even the recombinants 
RD1-pAP47 and RD1-pAP48, which overexpress intracellular ESAT-6, did not generate detectable ESAT-6 specific T-cell responses. Although antigen secretion is important for inducing immunity against $M$. tuberculosis, this is one of the first formal demonstrations of its importance. BCG, like M. tuberculosis, resides in the phagosome, where secreted antigens have ready access to the major histocompatibility complex class II antigen-processing pathway. This pathway is essential for inducing $\mathrm{CD}^{+} \mathrm{T}$ cells that produce IFN- $\gamma$, which is crucial for protection against tuberculosis. Further understanding of the mechanism of ESAT- 6 secretion could allow the development of BCG recombinants that deliver other antigens in the same way.

Our aim in this study was to qualitatively enhance the antigenicity of BCG. We used three distinct models to show that the recombinant secreting ESAT-6 and CFP-10 improved protection in spleens as compared with a BCG control. The lack of enhanced protection afforded to the lungs could be explained in a number of ways. Expression of the antigenic RD1 proteins by the recombinants might vary between organs. Alternatively, recruitment of RD1-specific effector $\mathrm{T}$ cells to the lungs could be less efficient than to the spleen. Also, the induction of immune responses against ESAT- 6 and CFP-10 might only restrict the dissemination of $M$. tuberculosis from the lung. Although BCG is known to inhibit disseminated forms of childhood tuberculosis, further reduction in the spread of $M$. tuberculosis from the lung could be an important attribute of a new vaccine. Primary tuberculosis occurs in the middle and lower lobes and is often acutely asymptomatic ${ }^{39}$. The bacteria need to reach the upper lobes, the most common site of disease, by spreading through the bloodstream. Therefore, a vaccine that inhibits dissemination of $M$. tuberculosis from the primary site of infection would probably have a major impact on the outcome of tuberculosis.

Recombinant BCG has advantages over other vaccines because it is inexpensive, easily produced and conveniently stored. Concerns remain, however, despite an enviable safety record; thus BCG is currently not administered to symptomatic HIV-infected individuals. We have previously shown that recombinant BCG RD1-2F9 grows more rapidly in severe combined immunodeficient mice, an extreme model of immunodeficiency, than does its parental BCG strain ${ }^{30}$. In both immunocompetent mice and guinea pigs, however, we observed only a slight increase in persistence and no increase in pathology. This may be beneficial, because the declining efficacy of BCG with serial passage has been attributed to an inadvertent increase in attenuation ${ }^{40}$. Ultimately, the robust enhancement in protection observed on re-incorporation of the RD1 locus is a compelling reason to include this genetic modification in any recombinant BCG vaccine, even if it may require a balancing attenuating mutation. The next stage will be to combine complementation with RD1 genes with the overproduction of existing ${ }^{23}$ or other missing immunodominant antigens of $M$. tuberculosis to determine how much BCG can be qualitatively and quantitatively improved.

\section{Methods}

Construction of recombinant BCG and $M$. microti vaccines. The RD11106, RD1-2F9 and pAP34 constructs have been previously described ${ }^{30}$. BCG Pasteur 1173P2 was from stocks held by G.M. at the Institut Pasteur. The PAP35 construct was created by excising an Afll fragment from RD12F9 (Fig. 1), effectively interrupting or removing all genes downstream of Rv3876. The pAP47 and pAP48 constructs were created by cloning PCRamplified esXA or es $X B A$ into the PGEM vector and then re-cloning in
pSM81 (supplied by S.V. Gordon, Surrey, UK) so that each gene was expressed from the $h s p 60$ promoter present in this shuttle vector. Electrotransformation and PCR screening of recombinants were done by standard procedures ${ }^{30}$.

Protein extraction and immunoblotting. Polyclonal antibodies against Rv3872, Rv3873 and CFP-10 and a monoclonal antibody against ESAT-6 have been described recently ${ }^{30}$. Cell-free protein extracts were prepared from early log-phase cultures grown in 7H9 or Sauton's media (Difco Laboratories, Sparks, Maryland) and processed by standard procedures. Proteins in supernatants from the same cultures were concentrated using a Millipore filter with a 3-kDa cutoff (Bedford, Massachusetts).

Immunological assays. Cultures of the recombinant vaccines were grown in parallel in $50 \mathrm{ml}$ of Middlebrook 7H9-ADC medium (Difco) supplemented with $0.05 \%$ Tween 80 and appropriate antibiotics. Bacteria were collected by low-speed centrifugation and washed once with $50 \mathrm{mM}$ sodium phosphate buffer $(\mathrm{pH} 7.0)$ before resuspension in $1-5 \mathrm{ml}$ of the same buffer. The bacteria were then sonicated briefly and allowed to stand for $2 \mathrm{~h}$ to allow residual aggregates to settle. The bacterial suspensions were divided into aliquots and frozen at $-80^{\circ} \mathrm{C}$. A single aliquot was defrosted for quantification of each vaccine lot. $\mathrm{C} 57 \mathrm{BL} / 6$ or $\mathrm{BALB} / \mathrm{c}$ mice were immunized subcutaneously or intravenously through the lateral tail vein with $10^{6} \mathrm{CFU}$ of recombinant vaccine. Three weeks later, single-cell suspensions of spleens were prepared in RPMI 1640, and $10^{6}$ cells each were plated onto 96-well flat-bottom plates in synthetic HL-1 medium (Bio-Whittaker, Walkersville, Maryland) complemented with $2 \mathrm{mM}$ L-glutamine, $100 \mathrm{IU} / \mathrm{ml}$ penicillin per $\mathrm{ml}$ and $100 \mathrm{~g}$ streptomycin per $\mathrm{ml}$ in the presence of various concentrations of antigens. After $3 \mathrm{~d}$ of incubation at $37^{\circ} \mathrm{C}$ with $5 \% \mathrm{CO}_{2}$, cell cultures were pulsed with $1 \mu \mathrm{Ci}$ methyl- $\left[{ }^{3} \mathrm{H}\right]$ thymidine for $16 \mathrm{~h}$. After collection, ${ }^{3} \mathrm{H}$ incorporation was measured with a Betaplate plate counter (Wallac, Turku, Finland). The phenotype of proliferating cells was determined by adding $1 \mu \mathrm{g} / \mathrm{ml}$ monoclonal antibodies against either CD4 (GK1.5) or CD8 (H35-17-2) to the cultures. To assay IFN- $\gamma$ production, splenocytes were stimulated with $10 \mu \mathrm{g} / \mathrm{ml}$ of PPD or synthetic peptides from ESAT-6 (amino acids 1-20), antigen 85A (amino acids 241-260), Mal-E (amino acids 100-114) or poliovirus type- 1 capsid protein VP1 (amino acids 103-116; refs. 41-43). After $72 \mathrm{~h}$ of culture, IFN- $\gamma$ was quantified in serial dilutions of supernatant by a sandwich ELISA (detection limit of $500 \mathrm{pg} / \mathrm{ml}$ ) using the monoclonal antibodies R4-6A2 and biotin-conjugated XMG1.2. A standard curve was obtained with recombinant mouse IFN- $\gamma$. Binding of the biotinylated monoclonal antibody was detected using horseradish peroxidase-coupled streptavidin with o-phenylenediamine as substrate. The $\mathrm{OD}_{492 \mathrm{~nm}}$ was read in a Labsystems Multiskan spectrophotometer (Labsystems, Cergy Pontoise, France).

Animal models. M. tuberculosis $\mathrm{H} 37 \mathrm{Rv}$ and the different recombinant vaccines were prepared in the same manner as for the immunological assays. Six-week-old female C57BL/6 mice (IFFA CREDO, Les Oncins, France) were vaccinated subcutaneously or intravenously with $10^{6} \mathrm{CFU}$, in both cases through the lateral tail vein. In certain experiments, mice were treated with antibiotics 2 months after immunization by adding isoniazid $(10 \mu \mathrm{g} / \mathrm{ml})$ and rifampicin $(20 \mu \mathrm{g} / \mathrm{ml})$ to drinking water for 3 weeks. Mice were then challenged either by the aerosol route or intravenously $\left(10^{6}\right.$ CFU). Mice were killed at different time points and organs were homogenized using a Mickle apparatus and 2.5- $\mathrm{mm}$ diameter glass beads. Serial 5 -fold dilutions in PBS were plated on 7H11 agar, and CFUs were ascertained after $2-3$ weeks of growth at $37^{\circ} \mathrm{C}$. Mean organ counts were analyzed statistically using ANOVA. For the guinea pig assays, groups of outbred female Dunkin-Hartley guinea pigs (David Hall, Burton-on-Trent, UK) were inoculated subcutaneously with $5 \times 10^{4} \mathrm{CFU}$. Guinea pigs were not treated with antibiotics before infection with M. tuberculosis. Aerosol challenge was performed 8 weeks after vaccination using a contained Henderson apparatus and an H37Rv (NCTC 7416) suspension to obtain an estimated retained inhaled dose of approximately $1,000 \mathrm{CFU} /$ lung $^{44,45}$. Organs were homogenized and dilutions plated out on $7 \mathrm{H} 11$ agar, as for the mouse experiments. Guinea pig experiments were carried out in the framework of the European Union Tuberculosis vaccine development program. All animal work was approved by institutional animal experimentation committees. 
Acknowledgments

We thank P. Chavarot, S.V. Gordon, M. Huerre, H. Khun, E. Maranghi, M. Marmiesse, $P$. Sebo and D. van Soolingen for advice, support, reagents and sharing of data. This work was funded by the Wellcome Trust, the Institut Pasteur (PTR110), the European Community (QLK2-CT1999-01093) and the Association Française Raoul Follereau. A.S.P. has a Wellcome Trust Training Fellowship in Clinical Tropical Medicine. This paper is dedicated to the memory of M.J. Colston.

\section{Competing interests statement}

The authors declare that they have no competing financial interests.

\section{RECEIVED 13 JANUARY; ACCEPTED 12 MARCH 2003}

1. Fine, P.E.M. Variation in protection by BCG: implications of and for heterologous immunity. Lancet 346, 1339-1345 (1995).

2. Brandt, L. et al. Failure of the Mycobacterium bovis BCG vaccine: some species of environmental mycobacteria block multiplication of BCG and induction of protective immunity to tuberculosis. Infect. Immun. 70, 672-678 (2002).

3. Harboe, M., Oettinger, T., Wiker, H.G., Rosenkrands, I. \& Andersen, P. Evidence for occurrence of the ESAT-6 protein in Mycobacterium tuberculosis and virulent Mycobacterium bovis and for its absence in Mycobacterium bovis BCG. Infect. Immun. 64, 16-22 (1996).

4. Behr, M.A. et al. Comparative genomics of BCG vaccines by whole-genome DNA microarrays. Science 284, 1520-1523 (1999).

5. Gordon, S.V. et al. Identification of variable regions in the genomes of tubercle bacilli using bacterial artificial chromosome arrays. Molec. Microbiol. 32, 643-656 (1999).

6. Mahairas, G.G., Sabo, P.J., Hickey, M.J., Singh, D.C. \& Stover, C.K. Molecular analysis of genetic differences between Mycobacterium bovis BCG and virulent M. bovis. J. Bacteriol. 178, 1274-1282 (1996).

7. Lewis, K.N. et al. Deletion of RD1 from Mycobacterium tuberculosis mimics bacille Calmette-Guérin attenuation. J. Infect. Dis. 187, 117-123 (2003).

8. Berthet, F.X., Rasmussen, P.B., Rosenkrandt, I., Andersen, P. \& Gicquel, B.A. Mycobacterium tuberculosis operon encoding ESAT-6 and a novel low-molecularmass culture filtrate protein (CFP-10). Microbiology 144, 3195-3203 (1998).

9. Colangelli, R. et al. MTSA-10, the product of the Rv3874 gene of Mycobacterium tuberculosis, elicits tuberculosis-specific, delayed-type hypersensitivity in guinea pigs. Infect. Immun. 68, 990-993 (2000).

10. Brandt, L., Oettinger, T., Holm, A. \& Anderson, P. Key epitopes on the ESAT-6 antigen recognized in mice during the recall of protective immunity to Mycobacterium tuberculosis. J. Immunol. 157, 3527-3533 (1996).

11. Sorensen, A.L., Nagai, S., Houen, G., Andersen, P. \& Andersen, A.B. Purification and characterization of a low-molecular-mass T-cell antigen secreted by Mycobacterium tuberculosis. Infect. Immun. 63, 1710-1717 (1995).

12. SkjØt, R.L.V. et al. Comparative evaluation of low-molecular-mass proteins from Mycobacterium tuberculosis identifies members of the ESAT- 6 family as immunodominant T-cell antigens. Infect. Immun. 68, 214-220 (2000).

13. Coler, R.N. et al. Vaccination with the T cell antigen Mtb 8.4 protects against challenge with Mycobacterium tuberculosis. J. Immunol. 166, 6227-6235 (2001).

14. Brandt, L., Elhay, M., Rosenkrands, I., Lindblad, E.B. \& Andersen, P. ESAT-6 subunit vaccination against Mycobacterium tuberculosis. Infect. Immun. 68, 791-795 (2000).

15. Brodin, P. et al. Bacterial artificial chromosome-based comparative genomic analysis identifies Mycobacterium microti as a natural ESAT-6 deletion mutant. Infect. Immun. 70, 5568-5578 (2002)

16. Hart, P.D. \& Sutherland, I. BCG and vole bacillus vaccines in the prevention of tuberculosis in adolescence and early adult life. Br. Med. J. 2, 293-295 (1977).

17. Sula, L. \& Radkovsky, J. Protective effects of M. microti vaccine against tuberculosis. J. Hyg. Epidemiol. Microbiol. Immunol. 20, 1-6 (1976).

18. Cole, S.T. et al. Deciphering the biology of Mycobacterium tuberculosis from the complete genome sequence. Nature 393, 537-544 (1998).

19. Tekaia, F. et al. Analysis of the proteome of Mycobacterium tuberculosis in silico. Tubercle Lung Dis. 79, 329-342 (1999).

20. Gey Van Pittius, N.C. et al. The ESAT-6 gene cluster of Mycobacterium tuberculosis and other high G+C Gram-positive bacteria. Genome Biol. 2, 44.1-44.18 (2001).
21. Baldwin, S.L. et al. Evaluation of new vaccines in the mouse and guinea pig model of tuberculosis. Infect. Immun. 66, 2951-2959 (1998).

22. Horwitz, M.A., Lee, B.W., Dillon, B.J. \& Harth, G. Protective immunity against tuberculosis induced by vaccination with major extracellular proteins of Mycobacterium tuberculosis. Proc. Natl. Acad. Sci. USA 92, 1530-1534 (1995).

23. Horwitz, M.A., Harth, G., Dillon, B.J. \& Maslesa-Galic, S. Recombinant Bacillus Calmette-Guerin (BCG) vaccines expressing the Mycobacterium tuberculosis 30-kDa major secretory protein induce greater protective immunity against tuberculosis than conventional BCG vaccines in a highly susceptible animal model. Proc. Natl. Acad. Sci. USA 97, 13853-13858 (2000).

24. Pallen, M.J. The ESAT-6/WXG100 superfamily — and a new Gram-positive secretion system? Trends Microbiol. 10, 209-212 (2002).

25. Neuwald, A.F., Aravind, L., Spouge, J.L. \& Koonin, E.V. AAA+: a class of chaperonelike ATPases associated with the assembly, operation, and disassembly of protein complexes. Genome Res. 9, 27-43 (1999).

26. Renshaw, P.S. et al. Conclusive evidence that the major T-cell antigens of the M. tuberculosis complex ESAT- 6 and CFP-10 form a tight, 1:1 complex and characterisation of the structural properties of ESAT-6, CFP-10 and the ESAT-6-CFP-10 complex: implications for pathogenesis and virulence. J. Biol. Chem. 277, 21598-21603 (2002).

27. Page, A.L. \& Parsot, C. Chaperones of the type III secretion pathway: jacks of all trades. Mol. Microbiol. 46, 1-11 (2002).

28. Brosch, R. et al. A new evolutionary scenario for the Mycobacterium tuberculosis complex. Proc. Natl. Acad. Sci. USA 99, 3684-3689 (2002)

29. Harboe, M. et al. B-cell epitopes and quantification of the ESAT-6 protein of Mycobacterium tuberculosis. Infect. Immun. 66, 717-723 (1998).

30. Pym, A.S., Brodin, P., Brosch, R., Huerre, M. \& Cole, S.T. Loss of RD1 contributed to the attenuation of the live tuberculosis vaccines Mycobacterium bovis BCG and Mycobacterium microti. Mol. Microbiol. 46, 709-717 (2002).

31. Lagranderie, M.R., Balazuc, A.M., Deriaud, E., Leclerc, C.D. \& Gheorghiu, M. Comparison of immune responses of mice immunized with five different Mycobacterium bovis BCG vaccine strains. Infect. Immun. 64, 1-9 (1996).

32. Kaufmann, S.H. How can immunology contribute to the control of tuberculosis? Nat. Rev. Immunol. 1, 20-30 (2001).

33. Young, D.B. \& Fruth, U. in New Generation Vaccines (eds. Levine, M., Woodrow, G. Kaper, J. \& Cobon G.S.) 631-645 (Marcel Dekker, New York, 1997).

34. Jackson, M. et al. Persistence and protective efficacy of a Mycobacterium tuberculosis auxotroph vaccine. Infect. Immun. 67, 2867-2873 (1999).

35. Hondalus, M.K. et al. Attenuation of and protection induced by a leucine auxotroph of Mycobacterium tuberculosis. Infect. Immun. 68, 2888-2898 (2000).

36. Brosch, R. et al. Use of a Mycobacterium tuberculosis H37Rv bacterial artificial chromosome (BAC) library for genome mapping, sequencing and comparative genomics. Infect. Immun. 66, 2221-2229 (1998).

37. Boesen, H., Jensen, B.N., Wilcke, T. \& Andersen, P. Human T-cell responses to secreted antigen fractions of Mycobacterium tuberculosis. Infect. Immun. 63, 1491-1497 (1995).

38. Finlay, B.B. \& Falkow, S. Common themes in microbial pathogenicity revisited Microbiol. Mol. Biol. Rev. 61, 136-169 (1997).

39. Garay, S.M. in Tuberculosis (eds. Rom, W.N. \& Garay, S.M.) 373-413 (Little, Brown and Company, Boston, 1996).

40. Behr, M.A. \& Small, P.M. Has BCG attenuated to impotence? Nature 389, 133-134 (1997).

41. Brandt, L. Key epitopes on the ESAT- 6 antigen recognized in mice during the recal of protective immunity to Mycobacterium tuberculosis. J. Immunol. 157, 3527-3533 (1996)

42. Huygen, K. et al. Mapping of $\mathrm{TH} 1$ helper T-cell epitopes on major secreted mycobacterial antigen 85A in mice infected with live Mycobacterium bovis BCG. Infect. Immun. 62, 363-370 (1994).

43. Leclerc, C., Deriaud, E., Mimic, V. \& Van der Werf, S. Identification of a T-cell epitope adjacent to neutralization antigenic site 1 of poliovirus type 1. J. Virol. 65, 711-718 (1991).

44. Williams, A., Davies, A., Marsh, P.D., Chambers, M.A. \& Hewinson, R.G. Comparison of the protective efficacy of bacille Calmette-Guérin vaccination against aerosol challenge with Mycobacterium tuberculosis and Mycobacterium bovis. Clin. Infect. Dis. 30 (suppl. 3), S299-S301 (2000).

45. Chambers, M.A. et al. Identification of a Mycobacterium bovis BCG auxotrophic mutant that protects guinea pigs against $M$. bovis and hematogenous spread of Mycobacterium tuberculosis without sensitization to tuberculin. Infect. Immun. 68 7094-7099 (2000). 